\title{
Feasibility of the cognitive assessment of nurs- ing-home residents with mild-to-moderate cogni- tive impairment using the intelligent voice-guided digital assistant THALIE: A pilot study
}

\begin{abstract}
Patricia Sagaspe $\mathrm{PhD}^{\mathrm{a}, \mathrm{b}, *}$, Véronique Lespinet-Najib $\mathrm{PhD}^{\mathrm{c}, \mathrm{d}}$, Sylvie Poulette $\mathrm{PhD}^{\mathrm{e}}$, Jean-Christophe Vasselon ${ }^{f}$, Amélie Roche $\mathrm{PhD}^{\mathrm{c}}$, Colette Fabrigoule $\mathrm{PhD}^{\mathrm{a}}$, Thierry Le Brun MD ${ }^{\mathrm{g}}$, Anne Bessous MD ${ }^{\mathrm{h}, \mathrm{i}}$, Emilie Fouchier ${ }^{\mathrm{g}}$, Camille Montagut ${ }^{\mathrm{h}}$, Morgane Ledoux ${ }^{i}$, André Joly PhD ${ }^{f}$, Pierre Philip MD PhD ${ }^{a, b}$

${ }^{a} \mathrm{CHU}$ de Bordeaux, Pôle Neurosciences Cliniques, F-33000 Bordeaux, France; bUniversité de Bordeaux, USR CNRS 3413 SANPSY Sommeil, Addiction et NeuroPSYChiatrie, F-33076 Bordeaux, France; 'UMR CNRS 5218 IMS Laboratoire de I'Intégration du Matériau au Système, Equipe Cognitique \& Ingénierie Humaine, F-33004 Talence, France; ${ }^{d}$ Ecole Nationale Supérieure de Cognitique ENSC, Bordeaux INP, F-33400 Talence, France; ${ }^{\text {SSylvie }}$ Poulette Conseil et Gestion de Projets, F-33370 Pompignac, France; ${ }^{f}$ SimSoft Healthcare, F-31670 Labège, France; gEHPAD La Mémoire des Ailes, F-33380 Marcheprime, France; ${ }^{h}$ EHPAD Résidence du Tertre, F-33126 Fronsac, France; ${ }^{i}$ EHPAD Les Mûriers, F-33360 Carignan-de-Bordeaux, France; *Corresponding author: patricia.sagaspe@chu-bordeaux.fr
\end{abstract}

\begin{abstract}
Background: Generally, little information is available on the cognitive status of institutionalized elderly persons. The intelligent voice-guided digital assistant THALIE is a computerbased device measuring memory, executive functions, language, visuo-spatial skills, and attention, developed to facilitate the cognitive assessment of residents in nursing homes.

Objective: The objective was to determine its feasibility of implementation for the clinical assessment of cognitive functioning in residents in nursing homes.

Methods: The computerized battery THALIE consisting of five neuropsychological tests and the traditional paper-and-pencil MMSE were administered to 14 elderly participants residing in three French nursing homes (EHPAD) who had mild-to-moderate cognitive impairment, potentially due to Alzheimer's disease or related disorders.

Non-parametric Spearman's correlations were performed to assess the association between THALIE and MMSE scores. Individual neuropsychological performance profiles were determined.

Results: THALIE performances were weakly correlated with MMSE score. THALIE gave a better overview per resident of the extent of impairment of their various cognitive functions than the MMSE used alone, and made it possible to identify less impaired functions. Conclusion: The feasibility of THALIE computerized cognitive assessment of residents in nursing homes is confirmed. It allows to evaluate various neurocognitive functions in nursing-home residents and holds much promise for setting up personalized cognitive care plans for these subjects.
\end{abstract}

Keywords: Older adults, cognitive impairment, computerized battery, nursing home

\section{INTRODUCTION}

The aging of the general population in western societies has progressively increased the number of people at risk of cognitive decline (Niu, Alvarez-Alvarez, Guillen-Grima, \& Aguinaga-Ontoso, 2017) and admission to a long-term care facility such as a nursing home (Gaugler, Yu, Krichbaum, \& Wyman, 2009). Cognitive decline, and dementia characterized by cognitive and behavioral functions leading to dependence for instrumental and basic activities of daily living, have been identified as major health care concerns among elderly nursing-home residents (Smith, Kokmen, \& O'Brien, 2000).

Many nursing-home residents have moderate-tosevere cognitive impairment and are diagnosed with dementia. A 22-year follow-up study based on the PAQUID French cohort ( $\mathrm{N}=3777$ community-dwelling people aged 65 years and older) suggested that the elderly living in institutions present greater cognitive decline than their community-dwelling counterparts (Gonzalez-Colaco Harmand et al., 2014). In France, however, little 
information is available on the cognitive status of institutionalized elderly persons.

The assessment and management of cognitive decline present a challenge for improving nursing-home care (Saliba et al., 2005). One of the roles of the psychologist in nursing homes is to assess the degree of residents' cognitive impairment with neuropsychological testing and to implement interventions based on cognitive and sensory stimulation.

While neuropsychological assessment reveals cognitive decline and improves the identification of individuals with dementia, traditional neuropsychological testing can be time-consuming and resource-demanding despite the relative brevity of each of them, so it is not feasible in all settings.

For these reasons, the neuropsychological test mainly used in nursing homes in France is the Mini-Mental State Examination (MMSE), a brief test recommended among others by a panel of experts (Haute Autorité de Santé (HAS), 2011). In the literature of aging research, some correlations were found between MMSE scores and performances in tests involving more complex functions such as memory and executive functions. A review (Tombaugh \& Mclntyre, 1992) showed the validity of the MMSE compared against tests that putatively identify and measure cognitive impairment such as the clock-drawing data. Moreover, studies found a significant correlation between MMSE scores and Trail-MakingTest scores (particularly with TMT-B speed score: $r=-0.54$ ) in elderly subjects (Olivera-Souza et al., 2000; Saint Martin, Sforza, Barthelemy, ThomasAnterion, \& Roche, 2012). In MCl patients, studies found significant correlations between MMSE scores and TMT (with TMT-B, $r=-.40$ ), and verbal fluency (animal naming) scores $(r=.38)$ (Jefferson et al., 2006; McDonnell et al., 2019). In a sample of the French Three-City Study $(n=1.516)$, the total MMSE scores and episodic memory evaluated by the Free and Cued Reminding Test (FCSRT) scores were also significantly correlated (with Total score: $r=.32$; Free score: $r=.40$ and Cued score: $r=.27$ ) (Carcaillon, Amieva, Auriacombe, Helmer, \& Dartigues, 2009). Regarding the relationship between the MMSE and cognitive functioning in non-demented elderly adults over 75 years of age, a study found that the predictive value of the MMSE is due to a subset of cognitively demanding items that are heavily influenced by both memory and visuospatial skills (Hill \& Backman, 1995). One of the limitations of the MMSE, is that it is not sufficient to accurately assess specific cognitive functions. Yet various cognitive domains including episodic memory, executive functions, language, visuo-spatial skills, and attention, need to be evaluated (Albert et al., 2011) since they are associated to activities of daily living (Barberger-Gateau, Fabrigoule, Rouch, Letenneur, \& Dartigues, 1999).

A new line of research is developing through the transfer of new technologies to the health sector, including cognitive evaluation and monitoring.

The use of computer technology is a promising solution to improve the quality of neuropsychological assessment (Parsey \& Schmitter-Edgecombe, 2013; Zygouris \& Tsolaki, 2015). The challenges are multifaceted such as improving cognitive evaluation when there are not enough specialized centers, alleviating mechanical tasks to improve clinical observation and the relationship with the patient, automating the time-consuming aspects (e.g., scoring) of tasks, facilitating the storage of data and patient follow-up and providing support when interpreting the findings.

Computerized neuropsychological batteries offer advantages over traditional paper-and-pencil tests such as ease and standardization of administration (Fillit, Simon, Doniger, \& Cummings, 2008; Schatz \& Browndyke, 2002; Zygouris \& Tsolaki, 2015). Several computerized test batteries have been developed for the cognitive evaluation or brief screening of older adults with mild cognitive impairment or dementia (for reviews, (Koo \& Vizer, 2019; Zygouris \& Tsolaki, 2015)).

To our knowledge, however, there is no available test battery designed for use in nursing homes in France. We have now developed the intelligent voice-guided digital assistant THALIE using an iterative user-centered approach in nursing homes for needs assessment, goal/task analysis, users interfaces design, and prototyping (Johnson, Johnson, \& Zhang, 2005).

THALIE is a computer-based device measuring some aspects of memory, executive functions, language, visuo-spatial skills, and attention. Five conventional neuropsychological tests were developed and incorporated into THALIE. In order to facilitate the cognitive assessment of residents in nursing homes, we developed this computerbased neuropsychological device. This study aimed to determine the feasibility of implementation of THALIE for the clinical assessment of cognitive functioning (memory, executive functions, language, visuo-spatial skills, and attention) in residents in nursing homes.

\section{Methods \\ Study population}

Residents, who were all volunteers, were recruited in three nursing homes (Etablissement d'Hébergement pour Personnes Agées Dépendantes, EHPAD) in the department of Gi- 
ronde, in France. They were clinically suspected of having mild-to-moderate cognitive impairment potentially due to Alzheimer's disease or related disorders.

The medical staff from each nursing home invited eligible patients to participate in the study.

Inclusion criteria were as follows:

(1) Having a Mini-Mental State Examination (MMSE) score $>10$

(2) Being at least 60 years old

(3) Benefiting from cognitive follow-up in the institution (MMSE)

(4) Not suffering from major depressive disorder according to clinical evaluation

All participants and/or legal representatives provided written informed consent, and the study was approved by the ethical committee (consultative committee for the protection of persons participating in biomedical research, Comité de Protection des Personnes [CPP] Sud-Est VI).

\section{Design}

A battery of neuropsychological tests was administered by trained neuropsychologists in each institution. First, participants were administered the traditional paper-and-pencil MMSE to evaluate global cognition (Hugonot-Diener, 2001). Second, they received a session lasting up to 60 minutes with THALIE. This session could be split over a period of up to 15 days. In parallel, usability and acceptability scales were completed by users (residents and the neuropsychologists) after each administration (not detailed here).

\section{Neuropsychological tests}

The neuropsychological battery comprised the following:

\section{A paper-and-pencil reference test}

The MMSE (Derouesné et al., 1999; Folstein, Folstein, \& McHugh, 1975; Hugonot-Diener, 2001) is one of the most widely used standardized neuropsychological tests for quantifying global cognitive functioning and cognitive change in clinical geriatric. It includes 30 items and assesses orientation, memory, attention, language, and praxis (Total score, range 0-30). Age, gender, and education-based normative data were established on a French population (Lechevallier-Michel, Fabrigoule, Lafont, Letenneur, \& Dartigues, 2004).

Five computerized tests from the THALIE software application

The Clock-Drawing Test (Sunderland et al., 1989; Wolf-Klein, Silverstone, Levy, \& Brod, 1989), which is commonly used in clinical assessment, is a simple and reliable test which draws upon memory, visuo-constructive and visuo-spatial abilities, and executive functions. A pre-drawn circle represents a clock face. The participant is required to put in the numbers so that it looks like a clock and then set the time to 10 minutes past 11 . There is no time limit. The maximum score is 7 points (presence, sequencing and position of numbers, and presence, placement, and size difference of hands). The time spent completing the test is also considered.

The 5-word test (5WT) (Dubois et al., 2002), which is a verbal memory test evaluating the ability to learn and recall a shortlist of five words presented with a semantic cue to control for memory encoding. The learning phase is followed by a recall consisting of retrieving the words, first spontaneously ("free recall"), and then with the help of a semantic cue ("cued recall") for those items not retrieved by a free recall. A five-minute interference nonverbal task (i.e., Clock-Drawing Test) separates the immediate and a delayed recall phase. It is a simple and reliable test for evaluating episodic memory in aging with sensitivity and specificity in identifying patients with Alzheimer's disease (AD) (Cowppli-Bony et al., 2005). It distinguishes between simple difficulties in the retrieval of stored information (facilitated by cuing) and genuine storage deficits characterizing typical Alzheimer's disease (not facilitated by cuing). The measures used were Total immediate recall (Sum of Free immediate recall and cued immediate recall) (range 0-5), Total delayed recall (Sum of Free delayed recall and cued delayed recall) (range 0-5), Total Score (Sum of Total immediate recall and Total delayed recall) (range 0-10) and Total Weighted Score (the weight of free recall scores is doubled compared to cued recall scores) (range 0-20).

The Trail-Making Test (TMT) (Tombaugh, 2004), which is a two-part neuropsychological test reflecting executive functioning. Participants are required to connect numbered circles in sequential order in the TMT-A, whereas they have to connect numbered and lettered circles in alternating numerical-alphabetical order in the TMTB (i.e., 1-A-2-B, etc.). TMT-A time is considered as a measure of processing speed, while TMT$\mathrm{B}$ time is viewed as an index of flexibility. The scores are the time (in seconds) needed to complete the sequence and the number of correct responses for Part $A$ and $B$, and perseverative errors (failure to alternate between number and letter sequences) for Part B. Age, gender, and education-based normative data were established on a French population (Amieva et al., 2009).

Zazzo's Cancellation Task (Zazzo, 1974), which measures selective attention and speed informa- 
Table 1. Socio-demographic and clinical characteristics of residents.

\begin{tabular}{lc}
\hline & Mean \pm SD or frequency \\
\hline Age (years) (range) & $91.04 \pm 3.38(84-97)$ \\
Gender (female) & $71.43 \%$ \\
Baccalaureate degree or higher & $35.71 \%$ \\
educational level & \\
Concomitant pathologies & \\
$\quad$ Diabetes & $15.38 \%$ \\
$\quad$ Hypertension & $46.15 \%$ \\
$\quad$ Depression - Anxiety & $23.08 \%$ \\
$\quad$ History of cardiovascular disease & $30.77 \%$ \\
Current medication use & $57.14 \%$ \\
$\quad$ Antidepressants & $28.57 \%$ \\
$\quad$ Benzodiazepines & $0 \%$ \\
$\quad$ Non-benzodiazepine anxiolytics & $19.50 \pm 5.06(13-29)$ \\
MMSE score (range) & $14.29 \%$ \\
MMSE score $\geq 24$ &
\end{tabular}

to familiarize themselves with it. A link to video tutorials and instructions/recommendations for use of THALIE is available on http://www.sanpsy.univ-bordeauxsegalen. fr/THALIE/

THALIE has two separate interfaces:

(1) An interface intended for patients on a tablet computer to visualize and perform tests in a multimodal way using a stylus on the touch screen and microphone. This interface allows tactile recording for graphomotor productions and voice recording for language productions.

(2) An interface intended for the professional that is displayed on a separate computer screen connected to a keyboard and a mouse to start the tests, to visualize on-screen instructions or run vocal instructions, to verify optional additional instructions, to monitor the progress of the test, to review or listen again to patients' productions, to facilitate immediate or delayed scoring of the results (qualitative and quantitative semi-automatic scoring system) and to print the synthesis of the results. Instructions are given in synthetic voice format through the computer's speakers. The clinician could activate or deactivate the voice guidance function as desired and take control of the test procedure if necessary.

\section{Statistical analyses}

Descriptive analyses

Quantitative variables were expressed as mean \pm standard deviation (SD), and qualitative variables were expressed as relative frequency.

\section{Comparative analyses}

Non-parametric correlations (Rho Spearman) with Bonferroni's adjusted level of significance were computed between the MMSE score and scores on the cognitive variables of THALIE.

\section{Individual profiles}

Each performance of residents on the cognitive tests (MMSE, Trail-Making-Test, Zazzo's Cancellation Task, and Isaacs Set Test) was classified according to normative data distributions established in a French population (Amieva, et al., 2009; Lechevallier-Michel, et al., 2004). The indicators classified performance from worse to better performance: lower percentile $(<10 \mathrm{P}$, 10th percentile), between 10th percentile and first quartile (10P-Q1), between first and second quartiles (Q1-Q2), between second and third quartiles (Q2-Q3), and highest quartile (>Q3, third quartile).

For the sake of convenience, percentile or quartile classifications for task completion times were reversed to match the lower percentile or quar- 
Table 2. Spearman correlations between cognitive variables scores for each THALIE tests and MMSE SCore in residents.

\begin{tabular}{|c|c|c|c|c|c|}
\hline $\begin{array}{l}\text { Correlations between cognitive variables for } \\
\text { THALIE tests and MMSE score }\end{array}$ & $\mathbf{N}$ & Mean \pm SD & $\begin{array}{c}\text { Rho } \\
\text { Spearman }\end{array}$ & $\begin{array}{c}\text { Exact } \\
\text { P value }\end{array}$ & $\begin{array}{l}\text { Bonferroni adjusted } \\
\text { level of significance }\end{array}$ \\
\hline \multicolumn{6}{|l|}{ Clock-Drawing Test } \\
\hline Total score & 10 & $4.3 \pm 2.6$ & -0.09 & NS & .025 \\
\hline Time & 10 & $116.7 \pm 53.9$ & 0.40 & NS & .025 \\
\hline \multicolumn{6}{|l|}{5 -word test (5WT) } \\
\hline Total immediate recall (Free+cued) & 10 & $4.4 \pm 0.8$ & 0.74 & $=.015$ & .0125 \\
\hline Total delayed recall (Free+cued) & 6 & $0.8 \pm 1.2$ & -0.69 & NS & .0125 \\
\hline Total Score (Immediate+delayed) & 6 & $5.3 \pm 1.4$ & 0.00 & NS & .0125 \\
\hline Total Weighted Score & 6 & $8.8 \pm 2.5$ & 0.12 & NS & .0125 \\
\hline \multicolumn{6}{|l|}{ Trail-Making Test (TMT) } \\
\hline TMT-A (correct responses) & 13 & $19.6 \pm 7.6$ & 0.31 & NS & .01 \\
\hline TMT-A (RTs) & 13 & $118.1 \pm 75.2$ & -0.10 & NS & .01 \\
\hline TMT-B (correct responses) & 12 & $7.7 \pm 8.0$ & 0.57 & NS & .01 \\
\hline TMT-B (RTs) & 12 & $225.5 \pm 141.1$ & 0.07 & NS & .01 \\
\hline TMT-B (perseverative errors) & 12 & $4.5 \pm 4.0$ & -0.58 & $=.049$ & .01 \\
\hline \multicolumn{6}{|l|}{ 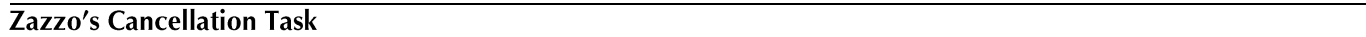 } \\
\hline Number of correct signs & 12 & $22.7 \pm 9.2$ & 0.75 & $=.005$ & .025 \\
\hline Time & 12 & $226.1 \pm 75.6$ & 0.46 & NS & .025 \\
\hline \multicolumn{6}{|l|}{ Isaacs Set Test } \\
\hline Number of correct responses $60 \mathrm{~s}$ & 13 & $40.7 \pm 16.9$ & 0.64 & $=.019$ & .016 \\
\hline Number of non-conscious repetitions $60 \mathrm{~s}$ & 13 & $7.1 \pm 5.2$ & -0.37 & NS & .016 \\
\hline Number of intrusions $60 \mathrm{~s}$ & 13 & $0.7 \pm 1.5$ & -0.01 & NS & .016 \\
\hline
\end{tabular}

tile with poor performance as for the classification of other cognitive variables.

Therefore, individual neuropsychological performance profiles were determined according to the performance on the different tests. TMTB completion task reaction times were not analyzed here because of the low number of correct responses and the possible premature interruption of the task.

\section{ResULTS}

Fourteen residents (10 females) were recruited (Table 1). All participants were right-handed. Regarding their legal status, two (14.29\%) were under guardianship. All participants had a normal or corrected vision and normal prehension. $85.71 \%$ of participants (12 out of 14 ) had a normal or corrected audition. $69.23 \%$ (9 out of 13 ) had more than one concomitant pathology and $42.86 \%$ (6 out of 14 ) had more than five concomitant treatments.

\section{Complete data for each THALIE test}

Percentages of complete data for each test recorded with THALIE were as follows: ClockDrawing Test: $71.43 \%$ (10 out of 14 ); 5 -Word Test (5WT): Immediate recall: $71.43 \%$ (10 out of 14) and Delayed recall: $42.86 \%$ (6 out of 14); Trail-Making Test (TMT) TMT-A: 92.86\% (13 out of 14) and TMT-B: $85.71 \%$ (12 out of 14); Zazzo test: $85.71 \%$ (12 out of 14) and Isaacs Set Test: $92.86 \%$ (13 out of 14$)$.

\section{Withdrawal from THALIE tests}

Percentages of withdrawal from each test recorded with THALIE were as follows: Clock-Drawing Test: $21.43 \%$ (3 out of 14 with 2 at the initiative of the neuropsychologist and 1 at the initiative of the resident, and because of motor and visual difficulties, and significant hand-tablet interference when the hand touched the surface of the tablet); 5WT: $7.14 \%$ ( 1 out of 14 at the initiative of the neuropsychologist because of significant anxiety of the resident); Trail-Making Test (TMT): $0 \%$; Zazzo test: $7.14 \%$ ( 1 out of 14 at the initiative of the resident because of visual difficulties) and Isaacs Set Test: 0\%. Five of the withdrawals concerned only 4 residents ( 2 withdrawals were observed for a particular resident on the ClockDrawing Test and the 5WT).

\section{Association between THALIE cognitive tests and MMSE}

Correlations between the MMSE and the THALIE cognitive tests were significant for the number of correct signs of Zazzo's Cancellation Task or exhibited a trend to significance for the Total immediate recall of 5-words Test and the number of correct responses of Isaacs Set Test (Table 2).

\section{Individual profiles of cognitive performances}

Table 3 shows that the worst performances $(<10 \mathrm{P}$ and 10P-Q1) were observed on the MMSE, on the TMT-B (i.e., correct responses and especially perseverative errors), and on completion time in Zazzo's cancellation task. Performances for other variables of THALIE tests (i.e., TMT-A correct responses, TMT-A completion time, Zazzo correct signs, Isaac Set Test correct responses) were more heterogeneous and contrasted between 


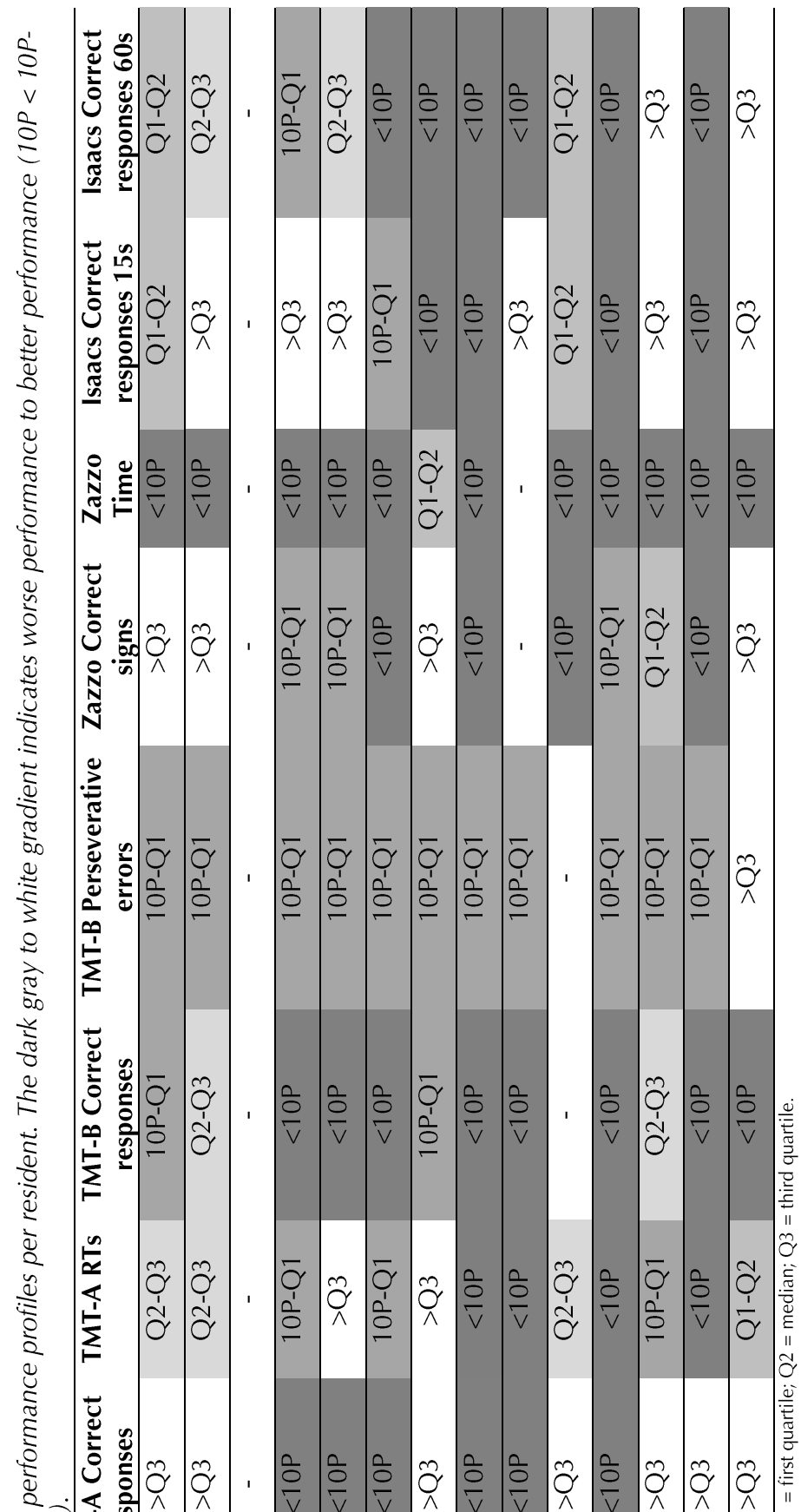

residents. Some residents had performances above the median (Q2-Q3) or even above the third quartile (>Q3), while others had low performances.

\section{Discussion}

With the increase in the number of elderly people at risk of cognitive decline (Niu, et al., 2017) and admission to a long-term care facility such as a nursing home (Gaugler, et al., 2009), the assessment and management of cognition present a challenge to improve nursing-home care (Saliba, et al., 2005).

Since neuropsychological testing can be costly and time-consuming, THALIE uses a set of emerging technologies to assist the clinician in the assessment of cognitive disorders in patients suffering from cognitive impairment.

This preliminary study demonstrates the feasibility of implementation and measurement using THALIE to evaluate cognition and memory (executive functions, visual-spatial skills, processing speed and cognitive flexibility, episodic memory) in nursinghome residents who scored 10 or more on the MMSE.

The percentage of complete data recorded for each THALIE test was acceptable (Clock-Drawing Test, 5WT, TMT, Zazzo, and Isaacs Set Test) and exceeded $70 \%$, except for delayed recall on the 5WT due to technical problems. Moreover, the oldest (age $\geq$ 84 years in our sample) patients did not refuse any THALIE neuropsychological test, and only five withdrawals occurred.

Few correlations were found between THALIE scores and the standard version of the MMSE scores in elderly nursing-home residents presenting mild-tomoderate cognitive impairment. These few correlations between THALIE scores and the MMSE scores could be due to a subset of cognitively demanding items in this latter that imply memory, 
visuospatial skills (Hill \& Backman, 1995), and executive components. Therefore, further studies will have to examine more precisely convergent validity of THALIE sub-tests with the paper-andpencil versions of the same tests or standard neuropsychological tests assessing the same domains.

Apart from these few correlations with the MMSE, THALIE results showed more contrasted patterns of performances. The MMSE score was low in all residents except two. While some residents performed poorly on the MMSE, their performance was better on some THALIE variables, thus highlighting the variability in the extent of their impaired cognitive functions. On the other hand, two residents who had higher MMSE scores performed less well on several THALIE variables. THALIE was informative even in good performers on the MMSE since it provided greater insight into the efficacy of their cognitive processes. These results underline the limitation of using the MMSE alone.

Overall, the most complex tests produced the most impaired performances, especially those involving processing speed and executive functions components. Scores were more consistently lower on the TMT-B (i.e., correct responses and especially perseverative errors) and completion time in Zazzo's cancellation task. The impaired TMT-B performances in most residents were expected because this test calls upon mental flexibility, which is known to be impaired early in the course of cognitive decline (Amieva et al., 1998). Regarding Zazzo's cancellation task, the speed component was more consistently impaired than the correct detection of signs per se. This result is in line with a previous study (Amieva, RouchLeroyer, Letenneur, Dartigues, \& Fabrigoule, 2004) showing that cognitive slowing is already present at a stage where target detection skills are not yet impaired in pre-demented subjects.

As expected, a wide variability on TMT-A and Isaacs Set Test performances was observed between residents, with some having preserved performances. The TMT-A is a simple test where numbers are linked in ascending order, a very automatic process. Regarding the Isaacs Set Test, the inter-individual variability in performances demonstrates the relative preservation of verbal fluency and semantic memory capacities in some residents.

This overall pattern of results underlines the limitation of using the MMSE alone and the contribution of testing the cognitive functions evaluated by THALIE (semantic memory, executive functions, attention, and processing speed) to gain a more precise overview of the impaired or relatively preserved capacities of subjects. This is crucial since memory, attention and executive functions are closely related to activities of daily living (Barberger-Gateau, et al., 1999; Vaughan \& Giovanello, 2010).

In conclusion, THALIE provides a finer analysis via individual profiles of the less altered cognitive processes that may then be the target of a personalized cognitive care plan within the institution.

Computerized batteries offer advantages over traditional paper-and-pencil neuropsychological tests (Fillit, et al., 2008; Parsey \& SchmitterEdgecombe, 2013; Schatz \& Browndyke, 2002; Zygouris \& Tsolaki, 2015) such as standardization of administration, thus limiting examiner bias and improving the reproducibility of testing. The automated and accurate capture of response parameters reveals the quantitative and qualitative aspects of the response, while the automatic scoring decreases the eventuality of rating errors. These automatic features promote interaction between the examiner and the patient by discharging the former of the "mechanical and systematic" aspects of administering tests and scoring. Automatic storage allows better data management, closer follow-up of individual performances over time, and more accurate transmission of information between health care staff. Finally, computer-based cognitive batteries seem less intimidating than the commonly used paperand-pencil neuropsychological tests (Gamaldo et al., 2018). These presumed assets will need to be verified in further studies, notably by studying user experience, quality of patient-clinician relationships, and saving time.

THALIE is therefore an innovative tool for the assessment and management of cognitive decline in nursing homes. However, its test-retest reliability needs to be studied to ensure the quality of patient follow-up. Therefore, additional studies in nursing homes with larger samples and a follow-up period are needed.

More broadly, THALIE could prove useful in memory clinic settings. Indeed, dementia remains largely underdiagnosed (Waldemar et al., 2007). A critical aspect of dementia is the early diagnosis (Haute Autorité de Santé (HAS), 2011), which provides an opportunity for interventions (Kivipelto, Mangialasche, \& Ngandu, 2018; Rodakowski, Saghafi, Butters, \& Skidmore, 2015). Further studies will have to demonstrate external validity of THALIE by its ability to distinguish healthy controls from cognitively impaired older adults (i.e., patients with $\mathrm{MCl}$ or $\mathrm{AD}$ ) or to distinguish between various degrees of cognitive impairment (i.e., patients with $\mathrm{MCl}$ versus $\mathrm{AD}$, or versus other types of dementia). 


\section{ConClusion}

To conclude, this study confirms that computerized cognitive assessment by THALIE is a feasible method for evaluating memory and other areas of cognitive functioning such as attention and executive functioning in elderly nursing-home residents. THALIE may enrich the evaluation of these cognitive components which are highly associated with activities of daily living and helps in setting up personalized care plans.

\section{Acknowledgements}

Special thanks go to the Thalie Project Consortium: SimSoft Healthcare, Labège; Agir Pour La Télémédecine (APLT) (notably Mickaël Chaleuil, Association President and Nathalie Limiati for providing administrative assistance), Sylvie Poulette, Conseil et Gestion de Projets, Pompignac; ITWELL (notably Catherine Argillier, General Manager, and Sarah Saint-Marc for providing administrative, technical and logistic assistance), Bordeaux; UMR CNRS 5218 - IMS, Talence; Bordeaux; USR CNRS 3413 SANPSY, Université de Bordeaux, Bordeaux; CHU de Bordeaux (notably Cécile Klochendler for financial management), Bordeaux.

A special thanks to Dr. Vincent Roth, General Manager Easy-CRF SAS, F-14920 Mathieu.

We also thank the students from the Ecole Nationale Supérieure de Cognitique ENSC, Bordeaux INP, Talence: Alexandra Pometko, Claire Dussard, Julien Doche and Elise Grevet for their work on the software manual based on video tutorials.

\section{Source Funding}

This project was supported by a grant from MSDAVENIR.

\section{Conflict of interest statement}

The authors report no disclosures relevant to the manuscript. They declare that the research was conducted in the absence of any commercial or financial relationships that could be construed as a potential conflict of interest.

\section{References}

Albert, M. S., DeKosky, S. T., Dickson, D., Dubois, B., Feldman, H. H., Fox, N. C., et al. (2011). The diagnosis of mild cognitive impairment due to Alzheimer's disease: recommendations from the National Institute on Aging-Alzheimer's Association workgroups on diagnostic guidelines for Alzheimer's disease. Alzheimers Dement, 7(3), 270-279.

Amieva, H., Lafont, S., Auriacombe, S., Rainville, C., Orgogozo, J. M., Dartigues, J. F., et al. (1998). Analysis of error types in the trial making test evidences an inhibitory deficit in dementia of the Alzheimer type. J Clin Exp Neuropsychol, 20(2), 280-285.

Amieva, H., Le Goff, M., Stoykova, R., Lafont, S., Ritchie, K., Tzourio, C., et al. (2009). Trail Making Test A et B (version sans correction des erreurs) : normes en population chez des sujets âgés, issues de l'étude des trois Cités. Revue de Neuropsychologie, 1, 210-220.

Amieva, H., Rouch-Leroyer, I., Letenneur, L., Dartigues, J. F., \& Fabrigoule, C. (2004). Cognitive slowing and learning of target detection skills in pre-demented subjects. Brain Cogn, 54(3), 212-214.

Barberger-Gateau, P., Fabrigoule, C., Rouch, I., Letenneur, L., \& Dartigues, J. F. (1999). Neuropsychological correlates of self-reported performance in instrumental activities of daily living and predic- tion of dementia. J Gerontol B Psychol Sci Soc Sci, 54(5), P293-303.

Carcaillon, L., Amieva, H., Auriacombe, S., Helmer, C., \& Dartigues, J. F. (2009). A subtest of the MMSE as a valid test of episodic memory? Comparison with the Free and Cued Reminding Test. Dement Geriatr Cogn Disord, 27(5), 429-438.

Cowppli-Bony, P., Fabrigoule, C., Letenneur, L., Ritchie, K., Alperovitch, A., Dartigues, J. F., et al. (2005). Le test des 5 mots : validité dans la detection de la maladie d'Alzheimer dans la population générale. Rev Neurol, 161(12 Pt 1), 1205-1212.

Derouesné, C., Poitreneau, J., Hugonot, L., Kalafat, M., Dubois, B., \& Laurent, B. (1999). Le Mini-Mental State Examination (MMSE) : un outil pratique pour l'evaluation de l'etat cognitif des patients par le clinicien. Version francaise consensuelle. Groupe de Recherche sur les Evaluations Cognitives (GRECO). Presse Med, 28(21), 1141-1148.

Dubois, B., Touchon, J., Portet, F., Ousset, P. J., Vellas, B., \& Michel, B. (2002). "Les 5 mots", épreuve simple et sensible pour le diagnostic de la maladie d'Alzheimer. Presse Med, 31(36), 1696-1699.

Fabrigoule, C., Rouch, I., Taberly, A., Letenneur, L., Commenges, D., Mazaux, J. M., et al. (1998). Cognitive process in preclinical phase of dementia. Brain, 121 ( Pt 1), 135-141.

Fillit, H. M., Simon, E. S., Doniger, G. M., \& Cummings, J. L. (2008). Practicality of a computerized system for cognitive assessment in the elderly. Alzheimers Dement, 4(1), 14-21.

Folstein, M. F., Folstein, S. E., \& McHugh, P. R. (1975). "Mini-mental state". A practical method for grading the cognitive state of patients for the clinician. J Psychiatr Res, 12(3), 189-198.

Gamaldo, A. A., Tan, S. C., Sardina, A. L., Henzi, C., Guest, R., Ross, L. A., et al. (2018). Older Black Adults' Satisfaction and Anxiety Levels After Completing Alternative Versus Traditional Cognitive Batteries. J Gerontol B Psychol Sci Soc Sci.

Gaugler, J. E., Yu, F., Krichbaum, K., \& Wyman, J. F. (2009). Predictors of nursing home admission for persons with dementia. Med Care, 47(2), 191-198.

Gonzalez-Colaco Harmand, M., Meillon, C., Rullier, L., Avila-Funes, J. A., Bergua, V., Dartigues, J. F., et al. (2014). Cognitive decline after entering a nursing home: a 22-year follow-up study of institutionalized and noninstitutionalized elderly people. J Am Med Dir Assoc, 15(7), 504-508.

Haute Autorité de Santé (HAS). (2011). Maladie d'Alzheimer et maladies apparentées : diagnostic et prise en charge. Recommandation de bonne pratique.

Hill, R. D., \& Backman, L. (1995). The relationship between the mini-mental state examination and cognitive functioning in normal elderly adults: a componential analysis. Age Ageing, 24(5), 440-446. 
Hugonot-Diener, L. (2001). MMS version consensuelle GRECO. In: La consultation en gériatrie. Paris: Masson; 2001. p. 13-20.

Isaacs, B., \& Kennie, A. T. (1973). The Set test as an aid to the detection of dementia in old people. $\mathrm{Br}$ J Psychiatry, 123(575), 467-470.

Jefferson, A. L., Wong, S., Bolen, E., Ozonoff, A., Green, R. C., \& Stern, R. A. (2006). Cognitive correlates of HVOT performance differ between individuals with mild cognitive impairment and normal controls. Arch Clin Neuropsychol, 21(5), 405-412.

Johnson, C. M., Johnson, T. R., \& Zhang, J. (2005). A user-centered framework for redesigning health care interfaces. J Biomed Inform, 38(1), 75-87.

Kivipelto, M., Mangialasche, F., \& Ngandu, T. (2018). Lifestyle interventions to prevent cognitive impairment, dementia and Alzheimer disease. Nat Rev Neurol, 14(11), 653-666.

Koo, B. M., \& Vizer, L. M. (2019). Mobile Technology for Cognitive Assessment of Older Adults: A Scoping Review. Innov Aging, 3(1), igy038.

Lechevallier-Michel, N., Fabrigoule, C., Lafont, S., Letenneur, L., \& Dartigues, J. F. (2004). Normes pour le MMSE, le test de retention visuelle de Benton, le set test d'Isaacs, le sous-test des codes de la WAIS et le test de barrage de Zazzo chez des sujets ages de 70 ans et plus : données de la cohorte PAQUID. Rev Neurol, 160(11), 1059-1070.

McDonnell, M., Dill, L., Panos, S., Amano, S., Brown, W., Giurgius, S., et al. (2019). Verbal fluency as a screening tool for mild cognitive impairment. Int Psychogeriatr, 1-8.

Niu, H., Alvarez-Alvarez, I., Guillen-Grima, F., \& Aguinaga-Ontoso, I. (2017). Prevalence and incidence of Alzheimer's disease in Europe: A meta-analysis. Neurologia, 32(8), 523-532.

Olivera-Souza, R. D., Moll, J., Passman, L. J., Cunha, F. C., Paes, F., Adriano, M. V., et al. (2000). Trail making and cognitive set-shifting. Arq Neuropsiquiatr, 58(3B), 826-829.

Parsey, C. M., \& Schmitter-Edgecombe, M. (2013). Applications of technology in neuropsychological assessment. Clin Neuropsychol, 27(8), 1328-1361.

Rodakowski, J., Saghafi, E., Butters, M. A., \& Skidmore, E. R. (2015). Non-pharmacological interventions for adults with mild cognitive impairment and early stage dementia: An updated scoping review. Mol
Aspects Med, 43-44, 38-53.

Saint Martin, M., Sforza, E., Barthelemy, J. C., ThomasAnterion, C., \& Roche, F. (2012). Does subjective sleep affect cognitive function in healthy elderly subjects? The Proof cohort. Sleep Med, 13(9), 1146-1152.

Saliba, D., Solomon, D., Rubenstein, L., Young, R., Schnelle, J., Roth, C., et al. (2005). Feasibility of quality indicators for the management of geriatric syndromes in nursing home residents. J Am Med Dir Assoc, 6(3 Suppl), S50-59.

Schatz, P., \& Browndyke, J. (2002). Applications of computer-based neuropsychological assessment. J Head Trauma Rehabil, 17(5), 395-410.

Smith, G. E., Kokmen, E., \& O'Brien, P. C. (2000). Risk factors for nursing home placement in a population-based dementia cohort. J Am Geriatr Soc, 48(5), 519-525.

Sunderland, T., Hill, J. L., Mellow, A. M., Lawlor, B. A., Gundersheimer, J., Newhouse, P. A., et al. (1989). Clock drawing in Alzheimer's disease. A novel measure of dementia severity. J Am Geriatr Soc, $37(8), 725-729$.

Tombaugh, T. N. (2004). Trail Making Test A and B: normative data stratified by age and education. Arch Clin Neuropsychol, 19(2), 203-214.

Tombaugh, T. N., \& Mclntyre, N. J. (1992). The minimental state examination: a comprehensive review. J Am Geriatr Soc, 40(9), 922-935.

Vaughan, L., \& Giovanello, K. (2010). Executive function in daily life: Age-related influences of executive processes on instrumental activities of daily living. Psychol Aging, 25(2), 343-355.

Waldemar, G., Phung, K. T., Burns, A., Georges, J., Hansen, F. R., Iliffe, S., et al. (2007). Access to diagnostic evaluation and treatment for dementia in Europe. Int J Geriatr Psychiatry, 22(1), 47-54.

Wolf-Klein, G. P., Silverstone, F. A., Levy, A. P., \& Brod, M. S. (1989). Screening for Alzheimer's disease by clock drawing. J Am Geriatr Soc, 37(8), 730-734.

Zazzo, R. (1974). Test des deux barrages. Actualités pédagogiques et psychologiques, Vol. 7. Neuchâtel : Delachaux et Nestlé.

Zygouris, S., \& Tsolaki, M. (2015). Computerized cognitive testing for older adults: a review. Am J Alzheimers Dis Other Demen, 30(1), 13-28. 